\title{
1 Disentangling the origins of virophages and polintons
}

3 Samuel Campbell ${ }^{1}$, Amr Aswad ${ }^{1}$, Aris Katzourakis*

Department of Zoology, University of Oxford, Oxford, OX1 3PS, UK

6

$7 \quad$ *Correspondence to: aris.katzourakis@zoo.ox.ac.uk

$8{ }^{1}$ These authors contributed equally to the work

10 Highlights

11 - Extensive horizontal gene transfer occurs in the eukaryote/giant

12 virus/virophage/transposon system.

13 - Virophages and polintons have a shared but obscured ancestry.

14 - We outline the evidence for a virus first origin for both polintons and virophages.

15 - Future studies that target narrower questions about specific genes or particular lineages will reveal more than overarching theories

17 - Paleovirological techniques can be used to test alternative hypotheses and uncover plausible

18 virophage-polinton intermediates

\section{Abstract}

21 Virophages and polintons are part of a complex system that also involves eukaryotes, giant 22 viruses, as well as other viruses and transposable elements. Virophages are cosmopolitan, 23 being found ranging from the Amazon River to Antarctic hypersaline lakes, while polintons 24 are found in many single celled and multicellular eukaryotes. Virophages and polintons have 25 a shared ancestry, but their exact origins are unknown and obscured by antiquity and extensive horizontal gene transfer. Paleovirology can help disentangle the complicated gene flow between these two, as well as their giant viral and eukaryotic hosts. We outline the evidence and theoretical support for polintons being descended from viruses and not vice versa. In order to disentangle the natural history of polintons and virophages, we suggest that there is much to be gained by embracing rigorous metagenomics and evolutionary analyses.

31 Methods from paleovirology will play a pivotal role in unravelling ancient relationships, 32 horizontal gene transfer and patterns of cross-species transmission. 


\section{Introduction}

40 The inhabitants of the microscopic world of giant viruses, their eukaryotic hosts, virophages, and various transposable elements (TEs) interact in a tangled web of interrelatedness, parasitism and coevolution. Giant viruses, can reach up to $1500 \mathrm{~nm}$ wide and harbour genomes over a megabase long. They are part of the nucleocytoplasmic large DNA viruses group (NCLDVs), that share a compliment of six 'core genes' [1-3], and includes families such as the Poxviridae and Phycodnaviridae. Several of the largest NCLDVs contain genes once considered exclusively cellular which has been taken as evidence in favour of a reduced-cell explanation for their gigantism [4]. However, genome expansion from smaller viruses via horizontal gene transfer (HGT) and genomic duplication is more consistent with the phylogenetic and genomic evidence, such as the presence of recently acquired host translational machinery in the Klosneuviruses [5]. Furthermore Poxviruses, which are also NCLDVs, are known to adaptively expand their genomes rapidly by up to $10 \%$, albeit only transiently [6].

Giant virus genomes have been colonised by TEs [7,8], and phylogenies reconstructed from different genes reveal conflicting topologies that indicating an extensive history of HGT. This complexity is reflective of their ecology, where giant viruses interact with both their hosts and associated satellite viruses known as virophages. All viruses are obligate parasites, but satellite viruses take parasitism a step further in that they require coinfection by a helper virus to replicate. The first virophage to be discovered, dubbed 'Sputnik', is associated with the giant virus Acanthamoeba castellanii mamavirus (ACMV) [9]. Like other giant viruses (and many NCLDVs), ACMV creates dense cytoplasmic structures known as viral factories. The term virophage is derived from bacteriophage, as Sputnik hijacks the replication machinery of these viral factories, rather than the native replication machinery of the host cell and has a negative impact on helper virus replication [9]. 
Virophages are dsDNA viruses but they do not appear to be closely related to other dsDNA viruses. Their morphogenesis genes, such as their pox-like A32 packaging proteins, place them in the diverse PRD1-adenovirus lineage [10], but some possess unrelated genes such as retroviral-like integrases, and they also contain genes of unknown function, some of which have no known homologues [11]. Interestingly however, there are remarkable similarities between virophages and a family of DNA transposable elements initially named mavericks but now more widely referred to as polintons [12]. Polintons are an atypical group of large TEs that are able to self-replicate using their own polymerase and integrase genes, hence the name 'Pol-Int-on' [13-15]. Although there are larger and smaller examples, most polintons are $\sim 15-22 \mathrm{kbp}$ in length $[16,17]$ which is comparable to that of virophage genomes. While virophages and polintons are extremely diverse, both at the nucleotide level and in terms of their genomic content, they nonetheless share multiple genes indicative of shared ancestry $[12,17,18]$.

The interactions between all the players in this tangled microscopic world are far from resolved, and the nature of the relationship between virophages and polintons is elusive. The clear indication of shared ancestry is set against a backdrop of frequent HGT that complicates attempts to identify distinct lineages, and the antiquity of their evolutionary interactions obscures the processes that shaped their combined natural histories $[16,18,19]$. However, because polintons are anciently integrated into host genomes, the relative evolutionary stability (in terms of mutation rate and frequency of HGT events) allows us to use techniques developed in paleovirology for the study of ancient viruses. As the field progresses and more data become available, we will be able to integrate observations from phylogenetic reconstruction and genomics. This will be informative for both virophages and polintons separately, but also in combined analyses that will reveal aspects of their shared evolutionary history. The synthesis of such analyses can additionally be contextualised with evidence from NCLDV evolutionary reconstruction and that of their eukaryotic hosts.

This paleovirological approach has previously been effective in resolving similarly complex evolutionary histories, such as that of a retrovirus with mammalian origins that has also integrated into farmed poultry, a herpesvirus, and poxvirus [20]. Similarly, a paleovirology approach was deployed to identify multiple gene transfer events between an unusual retroviral superantigen gene and three different herpesviruses, via two separate mammalian hosts [21]. In this paper, we review the prevailing evidence and opinions about the origins of 
virophages and polintons with a paleovirological perspective. We consider the extent to which

101 different aspects of their relatedness can be explained by their evolutionary interactions with

102 giant viruses and eukaryotic hosts.

103

\section{Virophages}

105 Since the discovery of Sputnik, other virophage families have been discovered, primarily in

106 metagenomic data [12,22-30]. Most virophages appear to have circular double stranded

107 DNA genomes ranging from $\sim 17-29 \mathrm{kbp}$, but the presence, order, and orientation of most

108 genes are not well conserved between proposed families. Only a fraction of virophages have

109 been experimentally validated and associated with a giant virus. Sputnik has been observed in

110 vivo, and its presence in viral factories results in dysfunctional and abortive ACMV virions.

111 This reduces the yield of ACMV by up to 70\% and decreases host cell lysis threefold [9].

112 Although distantly related to Sputnik, mavirus similarly inhibits its host giant virus Cafeteria

113 roenbergensis virus $(\mathrm{CroV})$ and increases C. roenbergensis survival [12]. The exact mechanisms

114 behind how Sputnik and mavirus hijack the viral factories are unknown, and since most

115 virophages are only known from metagenomic data, it is not certain how many of them

116 rescue the eukaryotic host. In contrast, the Zamilon virophage, a close relative of Sputnik,

117 does not inhibit APMV reproduction or cell lysis [26]. It is conceivable that like the subviral

118 satellite hepatitis D [31], a virophage could lead to increased morbidity and mortality in the

119 eukaryotic host. It also is unclear how other players in the system, such as TEs, affect the

120 outcome of the interaction between virophages, giant viruses, and eukaryotes.

\section{Polintons}

123 While giant viruses and virophages only infect single celled eukaryotes, polintons and

124 polinton-like elements (PLEs) have been described in a diverse array of single celled and

125 multicellular eukaryotes, from cnidarians to water moulds. Given how distantly related some

126 of the hosts are, and the notable absence of polintons in mammals and terrestrial plants

$127[16,17,32]$, their taxonomic distribution suggests frequent novel integrations, extensive HGT

128 between different kingdoms/super groups and the repeated loss in descendant lineages of an

129 ancient integration. Indeed, phylogenetic analysis indicates a mix of both vertical and

130 horizontal transmission [16]. This pattern is not unlike that of endogenous retrovirus

131 phylogenies, which contain both horizontally and vertically transmitted insertions [33]. As

132 paleovirology focuses on such questions of gene flow, it could be used to quantify the relative 
133 extent of these two processes via a statistical comparison of polinton and host phylogenies.

135 Virophages $\leftrightarrows$ Polintons

136 While convergent evolution and HGT could explain some of their similarities, extensive

137 phylogenetic and genomic evidence linking virophages and polintons makes a compelling

138 case for the hypothesis of shared ancestry, for which there is broad consensus [19,32,34].

139 However, this leads to questions on the directionality of gene flow and whether the ancestor

140 of both entities was originally a TE that resembles contemporary polintons, or if a viral

141 ancestor gave rise to virophages and their genomic relatives. Conclusive evidence for either

142 scenario has yet to be unearthed, and arguments for both have been proposed (Figure 1). In

143 the TE-first scenario, virophages are the descendants of 'escaped' polintons that recombined

144 with an unknown virus to acquire a capsid (Figure1A) [10]. An argument against this is that

145 because virophages are dependent on co-infection by giant viruses, while polintons are not,

146 the loss of dependency following integration is more readily explained than its arising de novo

147 in a DNA transposon [12,18]. Furthermore, there are seven genes in mavirus with similarity

148 to polinton genes, compared to only three potential homologs with other virophages like

149 Sputnik. This suggests that mavirus may be more closely related to polintons than it is to

150 other virophages, indicating that gene flow went from virus to TE, and that a virus is

151 ancestral to both groups [12,18,19]. 


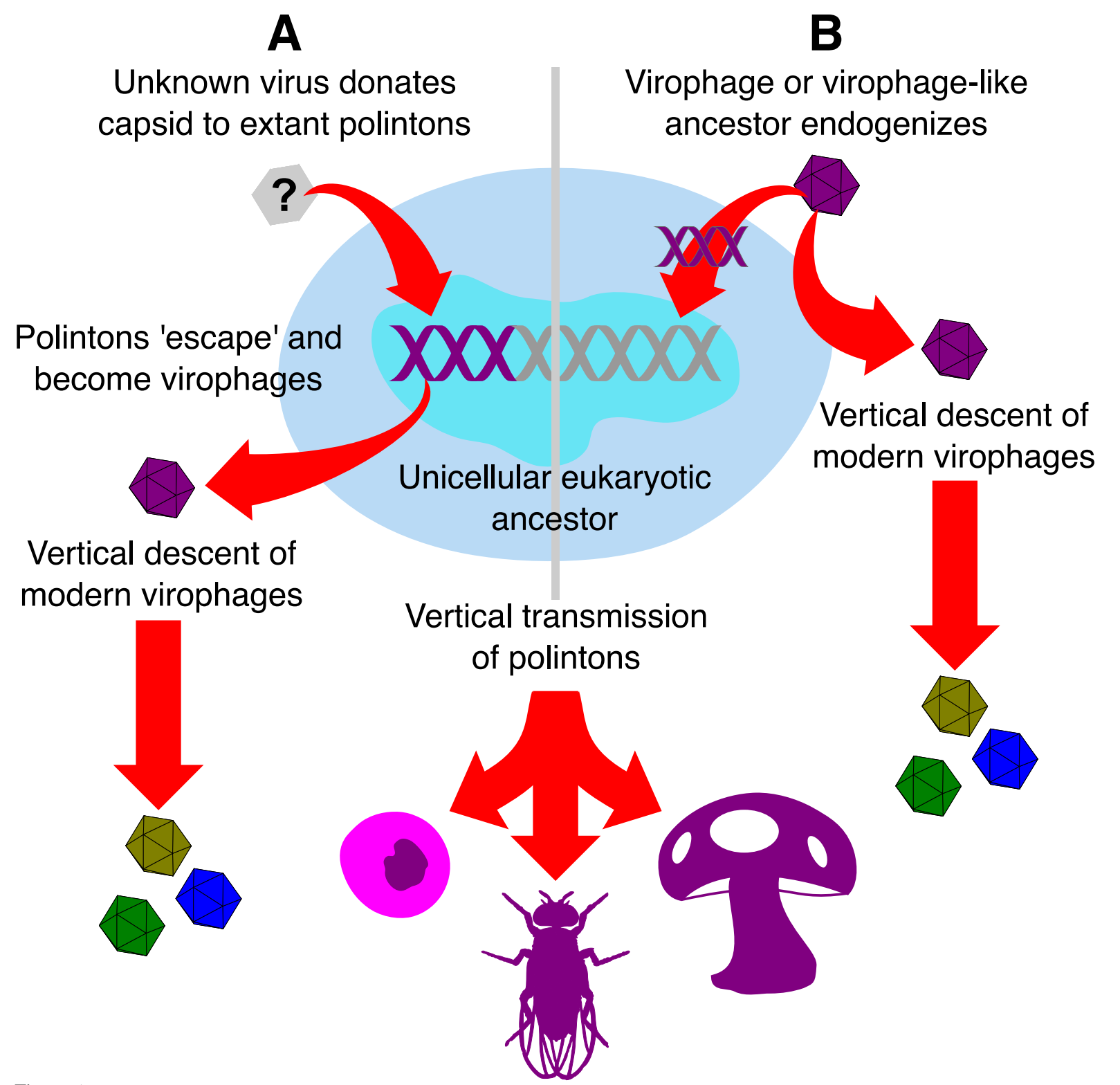

Figure 1.

Two proposed scenarios for the origins of polintons. A) An unknown virus (grey icosahedron) infected a cell and recombined with pre-existing polintons. The polintons then escaped as virophages (purple icosahedron). B) A virophage (purple

icosahedron) integrated into the genome of a cell and was then transmitted vertically, as a standard EVE, differentiating along with its hosts. In both cases polintons subsequently descend primarily vertically as do virophages.

156 Furthermore, there is experimental evidence of gene flow from a virophage to its host, which

157 supports the virophage-first hypothesis. The spontaneous integration of mavirus into $C$.

158 roenbergensis has been observed in vitro, where they remain quiescent until coinfection by GroV

159 results in the production and eventual release of mavirus [35]. Unlike with normal mavirus

160 infection, this does not seem to protect the individual cell initially infected with CroV, but it

161 does protect the wider population from lysis, suggesting that endogenous virophages can act as antiviral defence systems in clonal protist populations [35]. 

immunity (EDI) and has been described in over a dozen cases in multicellular organisms [36]. As well as experimental observations, transcriptionally active genes of putative virophage origin, dubbed virophage like elements (VLEs), are incorporated in the genome of the algae Bigelowiella natans [37]. No known giant virus has been observed parasitizing B. natans, so any defensive function is speculative. Nevertheless, VLEs could be indicative of a historical confrontation with an ancient giant virus [37], and techniques from paleovirology are wellplaced to investigating this. VLEs are in essence EVEs, and their presence gives us clues as to what sort of viruses ancestral B. natans had to contend with. It would be interesting to use functional paleovirology [38] to reconstitute virophages from VLEs to test their host giant virus range. Although they are the only two reported cases of virophage/VLE integration in eukaryotes, the cases of $B$. natans and $C$. roenbergensis provide evidence of an evolutionary scenario in which polintons arose from integrated virophages that were intitially evolutionarily maintained as EDIs $[35,37]$. However, even if some polintons arose from EDIs, others could have randomly integrated without substantial effect on the host, and drifted or Paleovirological discoveries of additional VLEs combined with evolutionary analysis of these integrated viral sequences can differentiate these two scenarios. With this amount of HGT, the questions must be examined at the level of individual genes. Designating 'core genes' in virophages $[10,19,40]$ will be a challenge, because the discovery of new taxa may whittle down their universality. Nonetheless, identifying widely conserved genes will facilitate answering specific questions, such as mapping the connections between polintons and virophages. For instance, integrase is going to be crucial as it is frequently found in both, even if we cannot include virophages where it is absent.

\section{Networks and Trees}

191 Different papers have presented phylogenetic trees with varying levels of disagreement,

192 depending on which genes are used and which taxa are being sampled - even the monophyly

193 of polintons and virophages is not consistent [12,19,29,32,41]. The matter is complicated

194 further by the fact that that virophages have open reading frames (ORFs) with no known non-

195 giant virus/virophage homologues [11]. Because of this, despite a plausible evolutionary

196 scenario for a 'virus first' model being demonstrated (supported with data and experimental 197 evidence $[37,42])$, the sequence of events in this chicken-and-egg scenario is unknown. 
199 Reconstructing the evolutionary history of virophages will be central to disentangling the 200 complex history of HGT between virophages, giant viruses, polintons, and their hosts. Their relatively small size and ability to integrate mean that virophages could facilitate HGT between giant viruses and their host eukaryotes. Genes taken from a giant virus by a virophage that is subsequently donated to a eukaryote could be viewed as 'vicarious EVEs' [18]. Indeed, genes of likely NCLDV origin have been discovered in land plants, despite there being no known exogenous NCLDVs that infect them [43,44]. The large size of the giant virus genomes would make integration of the entire viral genome without disrupting cellular functions difficult, which may explain why no giant viral EVEs have been identified. Nevertheless, large viral integrations can and do occur, such as herpesviruses in primates [45-47], phycodnaviruses in algae [48,49], as well as endogenized phycodnavirus segments [50,51].

211 One way to make sense of the tangled relationships between NCLDVs, eukaryotes,

212 virophages and bacteriophages is by drawing gene networks [10,19,34]. While these

213 accurately capture a general pattern of HGT between these entities, they provide limited

214 insight into the underlying biology, phylogenetic history, or direction of transfer.

215 Furthermore, these similarity-based approaches do not distinguish on the basis of gene

216 function. For example, many virophages contain genes not found in other virophage lineages

217 such as retroviral-like integrase in maviruses. Similarly, Sputnik appears evolutionarily linked

218 to bacterial TEs carrying TV-Pol family genes (e.g. IS605 or SCGmec elements) [52], and has

219 a phage-like bacterial transposase that is unrelated to integrases from mavirus, autonomous

220 polintons, and some PLEs [9]. Thus, in addition to recombination, this indicates convergent

221 evolution that gene network approaches cannot distinguish.

\section{Origins Of Virophages: Kicking The Can Down The Road}

224 If polintons are EVEs, descended from an anciently integrated virophage (Figure 1B), this does not answer where virophages came from. A third hypothesis addressing whether virophages or polintons came first puts hypothetical 'polintoviruses' at the origin of not just virophages and polintons, but also NCLDVs, bidnaviruses, and adenoviruses [10,53]. Based on the presence of ORFs that are predicted to form the double jelly roll fold seen in virophage capsid proteins, it has been suggested that many elements classified as non-viral transposable elements (i.e. polintons) are actually bona fide viruses [54]. In this hypothesis, tectiviruses (bacteriophages infecting gram-negative bacteria) came into eukaryotes with the 
capture of mitochondria [10,40]. Tectiviruses acquired cysteine protease and integrase from already extant transposons, and subsequently became polintoviruses, capable of existing exogenously and endogenously, while those that lost their capsid gene became intracellular polintons [40].

The polintoviruses hypothesis also suggests that these integrated tectiviruses escaped the nucleus via the acquisition of DNA dependent RNA polymerase and an mRNA capping system, forking into cytoplasmic plasmids via genome reduction and into NCLDVs via the replacement of POLB, and a 10-100 fold (or more) genome expansion [40]. Additionally, polintoviruses differentiated into other exogenous viruses, some of which began parasitizing and swapping genes with some NCLDVs [40].

244 Possession of a coat-protein encoding gene, as well the ability to form virions are the defining

245 features of a bona fide virus. Polintoviruses have never been observed, and thus far, there is no

246 indication that their putative capsid ORFs are anything more than endogenized remnants.

247 Nonetheless, it is likely that the ancestor of both polintons and virophages was a virus, and

248 future work remains to demonstrate what it may have resembled and to what extent it is also

249 the ancestor of other lineages.

250

251 In depth gene-by-gene paleovirological analysis will unravel the natural history

\section{2 of polintons and virophages}

253 Regardless of the direction of transfer, polintons are endogenous relatives of virophages. As

254 such, they can be studied under a paleovirology framework in the same manner as EVEs.

255 Over the last several decades, retroviruses have been systematically investigated using robust 256 phylogenetics and genome-wide statistical analyses. This has revealed insights ranging from 257 uncovering ancient patterns of cross-species transmissions across vertebrates [33,55-57], to 258 identifying the many ways that retroviral genes have been co-opted to serve a beneficial 259 function [58-60]. Moreover, the relationship between extant virophages and polintons is 260 analogous to that between contemporary and ancient retroviruses. For instance like polintons, 261 the diversity and range of ancient retroviruses far exceeds that of their contemporary cousins 262 [55]. Such paleovirological surveys have enabled us to re-evaluate our understanding of 263 groups like lentiviruses and foamy viruses, as well as identify the broad and influential effect they have on host genomes [58,60-62]. By applying the same paleovirology principles, we 
will be able to reach detailed and statistically robust conclusions about specific groups of polintons, virophages, and/or the genes contained within them.

Also central to the study of viruses in paleovirology is the adherence to certain standards of data analysis and rigorous evidence, since most research depends on third-party genomic data collected under different conditions and assembled to varying degrees of accuracy. The recognition that metagenomics and paleovirology have an overlapping set of methodologies and goals is giving rise to novel approaches in viral discovery and analysis [47,63]. Most virophages identified thus far have only bioinformatic representation, having been mined from large-scale metagenomic studies. While these sequences are invaluable, we must be cautious and apply the same level of scrutiny to these findings as we would with novel endogenous viruses. This includes offering supportive evidence for valid assembly as well as distinguishing between integrations and 'free-living' viruses.

\section{Conclusions}

There is unlikely to be a unifying theory that can at once explain the lack of synteny between virophages and giant viruses, between different lineages of virophages, and between virophages and polintons. Although a relationship between polintons and virophages is clear, there remains a large gap that separates the groups in terms of phylogenetic similarity, genomic organisation and gene content. Therefore while their ancestor was likely viral, the nature and identity of this virus remains to be determined. A more piecemeal approach that can explain parts of the puzzle with less uncertainty will inevitably develop as we identify new lineages of virophages and polintons. Moreover, increased taxonomic sampling will also reveal transitional viral fossils that could help disentangle the entwined natural histories of genes and genomes, by combining the information from phylogenetic reconstruction and analysis of genomic organisation.

\section{The application of techniques that synthesise analyses of both EVEs and viral genomes will} help reconstruct very ancient evolutionary events, and paleovirology is well equipped to deal with remarkably large timescales. For example, such hybrid approaches have been used to trace the origin of retroviruses to the seas of the early Palaeozoic Era more than 450 million years ago [62]. However, we must also be prepared to recognise that if the history of these entities extends far beyond such timescales - possibly even preceding the last universal cellular ancestor - then the answers to some (or all) of the questions posed here may be out of reach. 


\section{References and recommended reading}

301 Papers of particular interest, published within the period of review, have been highlighted as:

$302 *$ of special interest

$303 * *$ of outstanding interest

304

305 1. Iyer LLM, Balaji S, Koonin EE V., Aravind L: Evolutionary genomics of nucleo-

306 cytoplasmic large DNA viruses. Virus Res. 2006, 117:156-184.

307 2. Yutin N, Wolf Y, Raoult D, Koonin E: Eukaryotic large nucleo-cytoplasmic

308 DNA viruses: clusters of orthologous genes and reconstruction of viral 309 genome evolution. Virolf 2009, 6.

3103 . Yutin N, Koonin E: Pandoraviruses are highly derived phycodnaviruses.

311 DNA 2013, 8 .

312 4. Raoult D, Audic S, Robert C, Abergel C: The 1.2-megabase genome sequence

313 of Mimivirus. Science (80-. ). 2004, 306:1344-1350.

314 **5. Schulz F, Yutin N, Ivanova NN, Ortega DR, Lee TK, VierheiligJ, Daims H, Horn M, 315 Wagner M, Jensen GJ, et al.: Giant viruses with an expanded complement of 316 translation system components. Science (80-. ). 2017, 356:82-85.

$317 * *$ Describes a novel family of giant viruses with the most complete set of translational

318 machinery genes yet seen in viruses.

320 6. Elde NC, Child SJ, Eickbush MT, Kitzman JO, Rogers KS, Shendure J, Geballe AP, 321 Malik HS: Poxviruses deploy genomic accordions to adapt rapidly against 322 host antiviral defenses. Cell 2012, 150:831-41.

323 7. Desnues C, La Scola B, Yutin N, Fournous G, Robert C, Azza S, Jardot P, Monteil S, 324 Campocasso A, Koonin E V, et al.: Provirophages and transpovirons as the 325 diverse mobilome of giant viruses. PNAS 2012, 109:18078-83.

326 8. Sun C, Feschotte $\mathrm{C}, \mathrm{Wu}$ Z, Mueller RL: DNA transposons have colonized the 327 genome of the giant virus Pandoravirus salinus. BMC Biol. 2015, 13:38.

328 9. Scola B La, Desnues C, Pagnier I, Robert C: The virophage as a unique parasite of the giant mimivirus. Nature 2008, 455:100-104.

330 10. Krupovic M, Koonin E V: Polintons: a hotbed of eukaryotic virus, 
transposon and plasmid evolution. 2014, doi:10.1038/nrmicro3389.

332 11. Sun S, Scola B La, Bowman V: Structural studies of the Sputnik virophage. $\mathcal{F}$. Virol. 2010, 84:894-7.

334 12. Fischer M, Suttle C: A virophage at the origin of large DNA transposons. Science (80-. ). 2011, 332:231-234.

13. Feschotte $\mathrm{C}$, Pritham EJ: Non-mammalian c-integrases are encoded by giant transposable elements. Trends Genet. 2005, 21:551-552.

14. Pritham EJ, Putliwala T, Feschotte C: Mavericks, a novel class of giant transposable elements widespread in eukaryotes and related to DNA viruses. Gene 2007, 390:3-17.

15. Kapitonov V V., Jurka J: Self-synthesizing DNA transposons in eukaryotes. PNAS 2006, 103:4540-4545.

*17. W, Kojima KK, Kohany O: Repbase Update, a database of repetitive elements in eukaryotic genomes. Mob. DNA 2015, 6:11.

* The primary public repository of polinton sequences.

349 18. Katzourakis A, Aswad A: The origins of giant viruses, virophages and their relatives in host genomes. BMC Biol. 2014, 12:51.

19. Yutin N, Raoult D, Koonin E V: Virophages, polintons, and transpovirons: a complex evolutionary network of diverse selfish genetic elements with different reproduction strategies. Virol. F. 2013, 10:158.

20. Niewiadomska AM, Gifford RJ: The Extraordinary Evolutionary History of the Reticuloendotheliosis Viruses. PLoS Biol. 2013, 11:e1001642.

21. Aswad A, Katzourakis A: Convergent capture of retroviral superantigens by mammalian herpesviruses. Nat. Commun. 2015, 6:8299. Pfannkoch C, Lewis M, Hoffman JM, Gibson JA, et al.: Virophage control of antarctic algal host-virus dynamics. Proc. ... 2011, 108:6163-8.

23. Zhou J, Zhang W, Yan S, Xiao J, Zhang Y, Li B, Pan Y, Wang Y: Diversity of virophages in metagenomic data sets. F. Virol. 2013, 87:4225-36.

24. Gaia M, Pagnier I, Campocasso A, Fournous G, Raoult D, La Scola B: Broad 

Mimivirus Reporter. PLoS One 2013, 8:e61912.

25. Santini S, Jeudy S, Bartoli J, Poirot O, Lescot M, Abergel C, Barbe V, Wommack KE,

\section{*27. Bellas CM, Anesio AM, Barker G: Analysis of virus genomes from glacial}

\section{PgV-16T highlights the common ancestry of the largest known DNA}

26. Gaia M, Benamar S, Boughalmi M, Pagnier I, Croce O, Colson P, Raoult D, La Scola B: Zamilon, a Novel Virophage with Mimiviridae Host Specificity. PLoS One 2014, 9:e94923. environments reveals novel virus groups with unusual host interactions. Front. Microbiol. 2015, 6:656.

* Describes a metagenomically identified virophage from Greenland glacier showing homology in one of its genes to Pithovirus sibericum, a giant virus extracted from Siberian permafrost.

*28. Zhou J, Sun D, Childers A, McDermott TR, Wang Y, Liles MR: Three Novel Virophage Genomes Discovered from Yellowstone Lake Metagenomes. $\mathcal{F}$. Virol. 2015, 89:1278-1285.

* This paper expands on the number of virophages found in Yellowstone Lake, further indicating that multiple species of virophage can co-exist in a single environment.

*29. Gong C, Zhang W, Zhou X, Wang H, Sun G, Xiao J, Pan Y, Yan S, Wang Y: Novel Virophages Discovered in a Freshwater Lake in China. Front. Microbiol. 2016, 7:5.

* Metagenomically identifies a novel group of virophages via their major capsid proteins that are most closely related most Yellowstone Lake virophages as well Organic Lake Virophage, with some novel ORFs showing sequence similarity to (and possible horizontal gene transfer with) phycodnavirus ORFs.

*30. Oh S, Yoo D, Liu W-T: Metagenomics Reveals a Novel Virophage Population in a Tibetan Mountain Lake. Microbes Environ. 2016, 31:173-7.

* This paper metagenomically identifies numerous virophage like sequences closely related Organic Lake Virophage and Yellowstone Lake virophages as well as conclusively identifying a novel full virophage genome.

31. Fattovich G, Giustina G, Christensen E, Pantalena M, Zagni I, Realdi G, Schalm SW: Influence of hepatitis delta virus infection on morbidity and mortality in 
compensated cirrhosis type B. The European Concerted Action on Viral Hepatitis (Eurohep). Gut 2000, 46:420-6.

32. Yutin N, Shevchenko S, Kapitonov V, Krupovic M, Koonin E V.: A novel group of diverse Polinton-like viruses discovered by metagenome analysis. $B M C$ Biol. 2015, 13:95.

33. Hayward A, Grabherr M, Jern P: Broad-scale phylogenomics provides insights into retrovirus-host evolution. $P N A S$ 2013, 110:20146-51.

34. Iranzo J, Krupovic M, Koonin E V.: The Double-Stranded DNA Virosphere as a Modular Hierarchical Network of Gene Sharing. MBio 2016, 7:e00978-16.

**35. Fischer MG, Hackl T: Host genome integration and giant virus-induced reactivation of the virophage mavirus. Nature 2016, 540:288-291.

** Describes the population protective de novo integration of mavirus into $\mathrm{C}$. roenbergensis, a notes the similarity between the newly integrated provirophages and polintons.

36. Aswad, Amr, Katzourakis A: Paleovirology: The Study of Endogenous Viral Elements. In Virus Evolution: Current Research and Future Directions. Edited by Weaver SC, Denison M, Roossinck M, Vignuzzi M. Caister Academic Press; 2016:273-292.

37. Blanc G, Gallot-Lavallée L, Maumus F: Provirophages in the Bigelowiella genome bear testimony to past encounters with giant viruses. PNAS 2015, 112:E5318-5326.

** First report of transcriptionally active virophage like elements being found in a eukaryotic genome, presenting evidence for the integration of virophages into cellular genomes.

38. Katzourakis A: Paleovirology: inferring viral evolution from host genome sequence data. Phil Trans $R$ Soc B 2013, 368:20120493-.

39. Katzourakis A, Gifford RJ: Endogenous viral elements in animal genomes. PLoS Genet. 2010, 6:e1001191.

40. Krupovic M, Kuhn JH, Fischer MG: A classification system for virophages and satellite viruses. Arch. Virol. 2016, 161:233-247.

41. Yutin N, Kapitonov V V, Koonin E V: A new family of hybrid virophages from an animal gut metagenome. Biol. Direct 2015, 10:19.

42. Fischer MG, Hackl T: Genome Integration and Reactivation of the Virophage Mavirus In the Marine Protozoan Cafeteria roenbergensis. [date unknown], doi:10.1101/068312.

43. Maumus F, Epert A, Nogué F, Blanc G: Plant genomes enclose footprints of past infections by giant virus relatives. Nat. Commun. 2014, 5:4268. 
*44. Mushegian A, Shipunov A, Elena SF: Changes in the composition of the RNA virome mark evolutionary transitions in green plants. BMC Biol. 2016, 14:68.

* This paper indicates that there was a transition to smaller viruses during the colonisation of land by plants which may give clues as to why known giant viruses and virophages only infect single celled eukaryotes.

45. Luppi M, Marasca R, Barozzi P, Ferrari S, Ceccherini-Nelli L, Batoni G, Merelli E, Torelli G: Three cases of human herpesvirus-6 latent infection: integration of viral genome in peripheral blood mononuclear cell DNA. 7 . Med. Virol. 1993, 40:44-52.

46. Morissette $G$, Flamand L: Herpesviruses and chromosomal integration. $\mathcal{F}$. Virol. 2010, 84:12100-9.

47. Aswad A, Katzourakis A: The first endogenous herpesvirus, identified in the tarsier genome, and novel sequences from primate rhadinoviruses and lymphocryptoviruses. PLoS Genet. 2014, 10:e1004332.

48. Delaroque N, Maier I, Knippers R, Müller DG: Persistent virus integration into the genome of its algal host, Ectocarpus siliculosus (Phaeophyceae). F. Gen. Virol. 1999, 80 ( Pt 6):1367-70.

49. Meints RH, Ivey RG, Lee AM, Choi T-J: Identification of Two Virus Integration Sites in the Brown Alga Feldmannia Chromosome. F. Virol. 2008, 82:1407-1413.

50. Blanc G, Duncan G, Agarkova I, Borodovsky M, Gurnon J, Kuo A, Lindquist E, Lucas S, Pangilinan J, Polle J, et al.: The Chlorella variabilis NC64A genome reveals adaptation to photosymbiosis, coevolution with viruses, and cryptic sex. Plant Cell 2010, 22:2943-55.

51. Read BA, KegelJ, Klute MJ, Kuo A, Lefebvre SC, Maumus F, Mayer C, Miller J, Monier A, Salamov A, et al.: Pan genome of the phytoplankton Emiliania underpins its global distribution. Nature 2013, 499:209-13.

52. Iyer LM, Abhiman S, Aravind L: A new family of polymerases related to superfamily A DNA polymerases and T7-like DNA-dependent RNA polymerases. Biol. Direct 2008, 3:39.

*53. Koonin E V., Krupovic M, Yutin N: Evolution of double-stranded DNA viruses of eukaryotes: from bacteriophages to transposons to giant viruses. Ann. $\mathcal{N}$. r. Acad. Sci. 2015, 1341:10-24. 
* Proposes an evolutionary relationship between hypothetical polintoviruses and adenoviruses, bidnaviruses, NCLDVs, polintons. tectivirus bacteriophages, various plasmids, and virophages.

54. Krupovic M, Bamford DH, Koonin E V: Conservation of major and minor jelly-roll capsid proteins in Polinton (Maverick) transposons suggests that they are bona fide viruses. Biol. Direct 2014, 9:6.

55. Hayward A, Cornwallis CK, Jern P: Pan-vertebrate comparative genomics unmasks retrovirus macroevolution. PNAS 2014, 112:464-9.

56. Gui J, Tachedjian G, Wang L-F: Bats and Rodents Shape Mammalian Retroviral Phylogeny. Sci. Rep. 2015, 5:16561.

57. Diehl WE, Patel N, Halm K, Johnson WE: Tracking interspecies transmission and long-term evolution of an ancient retrovirus using the genomes of modern mammals | eLife Lens. Elife 2016, 5:e12704.

58. Aswad A, Katzourakis A: Paleovirology and virally derived immunity. Trends Ecol. Evol. 2012, 27:627-636.

59. Dupressoir A, Vernochet C, Bawa O, Harper F, Pierron G, Opolon P, Heidmann T: Syncytin-A knockout mice demonstrate the critical role in placentation of a fusogenic, endogenous retrovirus-derived, envelope gene. $P \mathcal{N} A S$ 2009, 106: $12127-32$.

60. Chuong EB, Elde NC, Feschotte C: Regulatory evolution of innate immunity through co-option of endogenous retroviruses. Science (80-. ). 2016, 351:10831087.

61. Katzourakis A, Gifford RJ, Tristem M, Gilbert MTP, Pybus OG: Macroevolution of complex retroviruses. Science 2009, 325:1512.

**62. Aiewsakun P, Katzourakis A: Marine origin of retroviruses in the early Palaeozoic Era. Nat. Commun. 2017, 8:13954.

** Describes for the first time the origin of retroviruses $>450$ million years ago demonstrating the utility of paleovirology in deciphering the origins of ancient lineages.

63. Houldcroft CJ, Breuer J: Tales from the crypt and coral reef: the successes and challenges of identifying new herpesviruses using metagenomics. Front. Microbiol. 2015, 6:188. 